\title{
Médiation, médiatisation et instruments d'enseignement : du triangle au « carré pédagogique »
}

Joseph Rézeau

\section{(2) OpenEdition}

\section{Journals}

Édition électronique

URL : http://journals.openedition.org/asp/1656

DOI : 10.4000/asp. 1656

ISBN : 978-2-8218-0388-6

ISSN : 2108-6354

\section{Éditeur}

Groupe d'étude et de recherche en anglais de spécialité

\section{Édition imprimée}

Date de publication : 1 décembre 2002

Pagination : 183-200

ISSN : 1246-8185

\section{Référence électronique}

Joseph Rézeau, «Médiation, médiatisation et instruments d'enseignement : du triangle au « carré pédagogique » », ASp [En ligne], 35-36 | 2002, mis en ligne le 28 août 2010, consulté le 21 décembre 2020. URL : http://journals.openedition.org/asp/1656 ; DOI : https://doi.org/10.4000/asp.1656

Ce document a été généré automatiquement le 21 décembre 2020.

Tous droits réservés 


\title{
Médiation, médiatisation et instruments d'enseignement : du triangle au « carré pédagogique »
}

\author{
Joseph Rézeau
}

\section{Introduction}

1 Le thème de la médiation proposé pour le $22^{\mathrm{e}}$ colloque du GERAS est au cœur d'une recherche que nous menons depuis bientôt six ans dans le cadre d'un enseignement de type LANSAD ${ }^{1}$ à l'université de Rennes 2 . Cette recherche s'est concrétisée dans une thèse soutenue à Bordeaux en décembre 2001 et intitulée : « Médiatisation et médiation dans l'enseignement des langues en environnement multimédia. Le cas de l'apprentissage de l'anglais en DEUG d'Histoire de l'art à l'université "².

2 Nous résumerons ici l'essentiel de la démarche théorique qui nous a amené à définir et à situer les concepts de médiation et de médiatisation dans le cadre de la situation pédagogique. Il s'agira tout d'abord de revisiter le modèle classique du triangle pédagogique pour y redéfinir la place du processus Enseigner. Dans un deuxième point, nous essaierons de définir et de situer la médiation pédagogique. La recherche entreprise faisant appel aux nouvelles technologies du multimédia, il s'agira ensuite d'explorer les relations entre médiatisation et médiation. Enfin, nous proposerons de réconcilier médiation et médiatisation dans la « nouvelle médiation pédagogique ». 


\section{Du triangle pédagogique à la médiation pédagogique}

\subsection{Le triangle pédagogique de Houssaye}

Le triangle pédagogique est discuté par Jean Houssaye (1988) dans son ouvrage désormais classique. La thèse de cet auteur est la suivante :

4 La situation pédagogique peut être définie comme un triangle composé de trois éléments, le savoir, le professeur et les élèves, dont deux se constituent comme sujets tandis que le troisième doit accepter la place du mort ou, à défaut, se mettre à faire le fou. Les processus sont au nombre de trois :

- « enseigner » qui privilégie l'axe professeur-savoir

- «former » qui privilégie l'axe professeur-élèves

- « apprendre » qui privilégie l'axe élèves-savoir.

Figure 1. Le triangle pédagogique de J. Houssaye

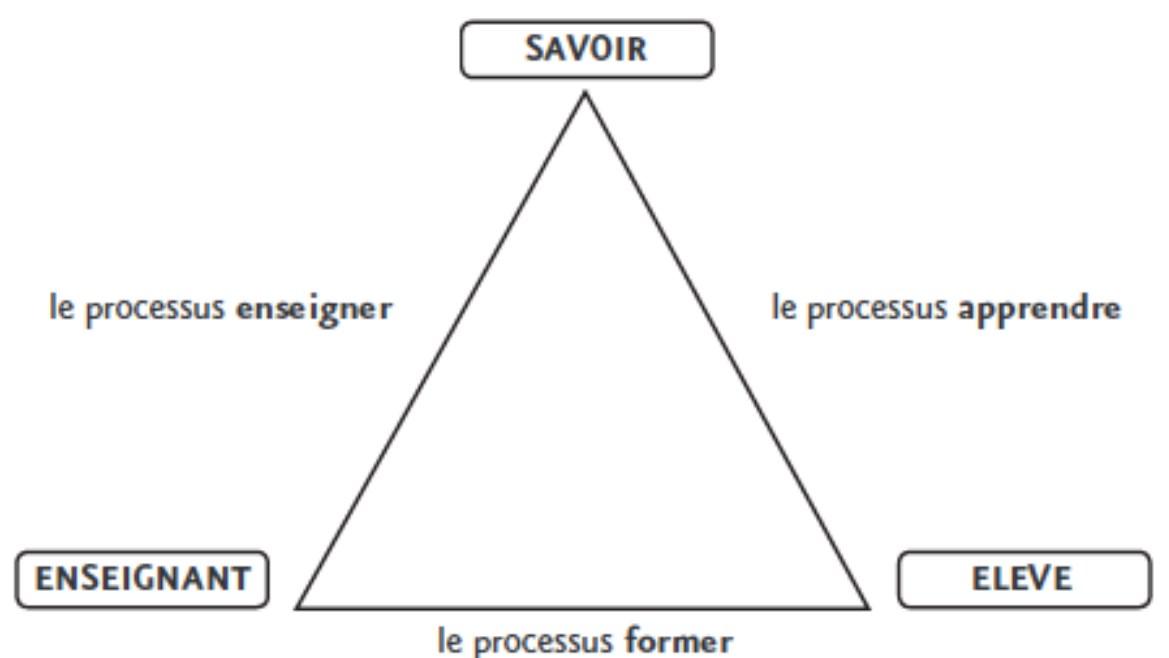

5 Pour Houssaye, seul le processus Enseigner (qu'il associe aux pédagogies traditionnelles centrées sur le contenu) exclut toute médiation. Les pédagogies libertaires et nondirectives, qui relèvent $\mathrm{du}$ processus «former», instituent l'enseignant comme médiateur. Ces pédagogies ont leurs racines dans les mouvements de l'École active et de l'Éducation nouvelle, chez des pédagogues comme Dewey et Freinet, dans la pédagogie non-directive de Carl Rogers, etc. Quant aux pédagogies qui mettent l'accent sur le processus « apprendre », « l'individu ou le groupe y deviennent leurs propres médiateurs dans l'accès au savoir» $(1988: 178)$. On peut cependant s'interroger sur la possibilité d'existence d'une auto-médiation...

\subsection{Le modèle SOMA}

6 Germain (1989) a adapté à la didactique des langues étrangères le modèle de la situation pédagogique de Legendre (1988). On trouve dans ce modèle quatre composantes : l'objet (le savoir, c'est-à-dire langue et culture), le Sujet (l'apprenant de L2), l'Agent (moyens 
humains et non-humains) et le Milieu, et trois relations: d'enseignement (A-S), d'apprentissage (S-O) et didactique (A-O) (voir figure 2).

Figure 2. Le modèle SOMA de la situation pédagogique (Legendre 1988, adapté à la didactique des langues par Germain 1989)

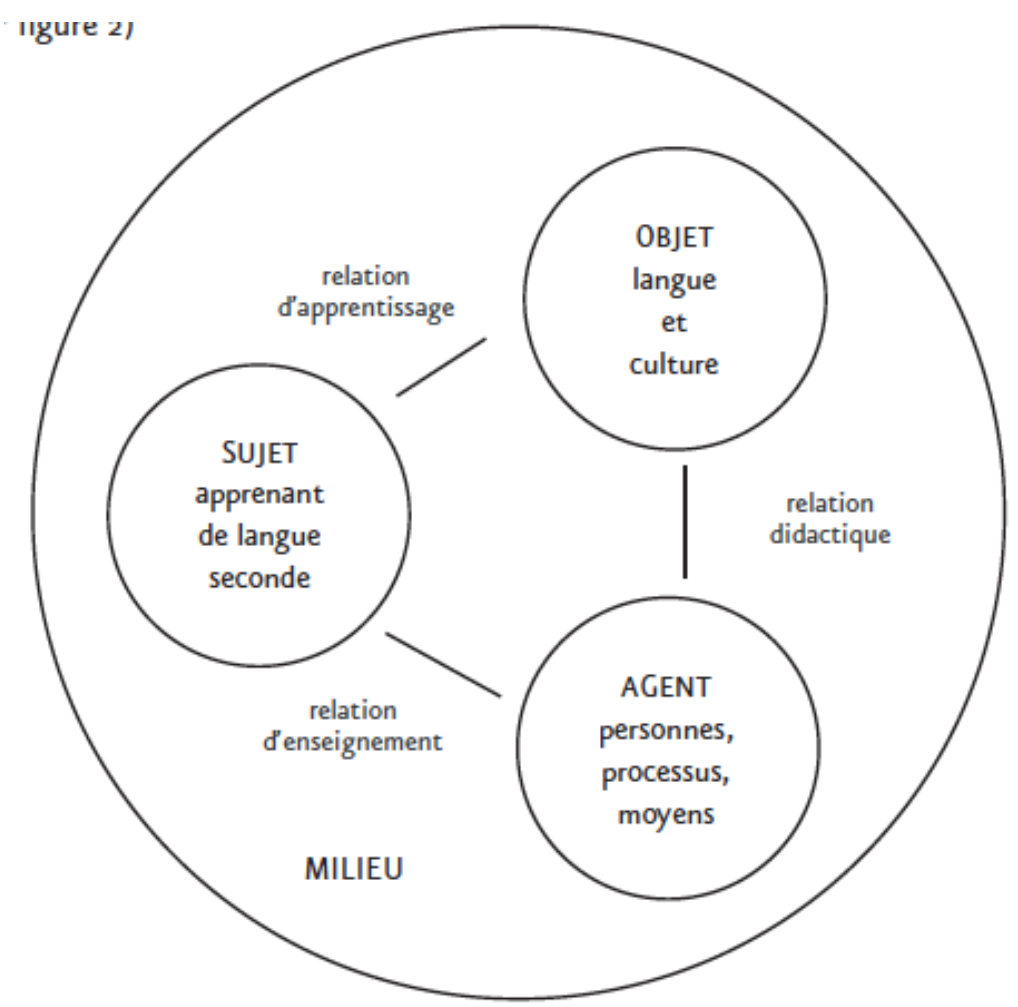

7 On pourrait multiplier les exemples de modèles de la situation d'enseignementapprentissage, mais le simple examen des deux modèles présentés ici suffit à démontrer qu'il n'a pas de consensus sur la place à attribuer au processus Enseigner : il est tantôt placé sur l'axe enseignant-savoir (chez Houssaye) tantôt sur l'axe Élève-Enseignant (chez Legendre et Germain).

Nous avons élaboré un premier modèle, basé sur le triangle pédagogique de Houssaye, de qui nous avons conservé la terminologie des trois « processus » : Apprendre, Enseigner et Former. Nous y intégrons le champ de la didactique, que nous associons à la gestion de l'information et celui de la pédagogie, qui relève de « l'économie de la médiation ou de la communication » (d'après Lerbert 1984 : 33). Pour faire pendant aux deux champs de la didactique et de la pédagogie, nous proposons, à défaut d'oser le néologisme d'« apprentique », mais pour tout du moins mettre en évidence le point de vue émique de notre approche, « l'art de l'apprentissage ».

9 Ce premier modèle permet d'étudier séparément (en les opposant) deux rôles de l'enseignant: le didacticien d'une part et le pédagogue de l'autre. Ou encore, pour reprendre les termes de Danièle Bailly, la réflexion et la recherche d'une part et la mise en œuvre pratique de l'autre. Mais il n'est pas satisfaisant en ce qu'il ne permet pas de définir le processus Enseigner, qui semble avoir été évacué du triangle pédagogique.

C'est pourquoi nous proposons - dans un deuxième temps - d'attribuer au processus Enseigner une place à part entière dans le triangle pédagogique, en le considérant comme la résultante des processus «former » et «didactiser ». Il apparaitt en effet que, s'il y a 
bien un processus «former " qui trouve naturellement sa place sur l'axe enseignantapprenant du triangle (on forme quelqu'un), c'est restreindre la portée du processus Enseigner que de le placer sur l'axe enseignant-savoir (comme le fait Houssaye). On enseigne bien quelque chose (un savoir), mais on enseigne toujours ce savoir à quelqu'un.

Figure 3. Deuxième modèle de la situation d'enseignement-apprentissage

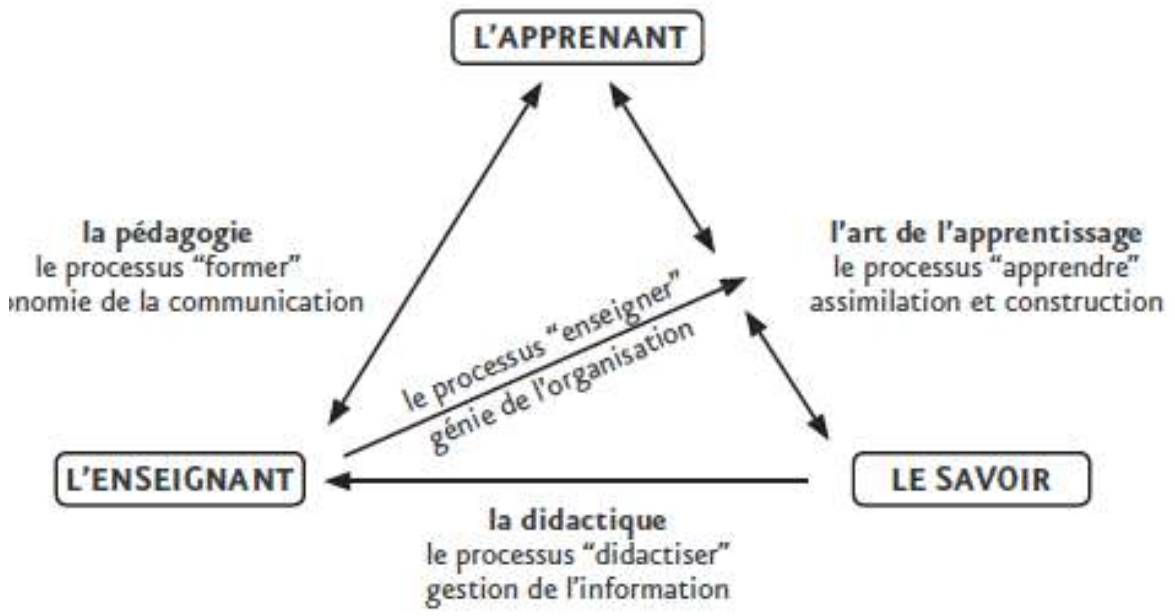

11 Cette nouvelle configuration offre en outre l'avantage de faire coïncider la topologie de la représentation du concept Enseigner avec ce que nous considérons comme sa fonction essentielle, celle de la médiation : sur le triangle pédagogique, le processus Enseigner est en position de médiane et de médiatrice. Maintenant que le processus Enseigner se trouve clairement placé en position médiatrice entre l'apprenant et le savoir, nous nous proposons d'explorer le concept de médiation en général et de médiation pédagogique en particulier.

\section{La médiation pédagogique}

\subsection{Quelques définitions}

Le dictionnaire Grand Robert électronique (GRE) définit ainsi le mot médiation :

1. (1561). Entremise destinée à mettre d'accord, à concilier ou à réconcilier des personnes, des partis... - Arbitrage, conciliation, entremise, intermédiaire, intervention. 2. [...] - Philos. Processus créateur par lequel on passe d'un terme initial à un terme final (dans la dialectique de Hegel, de Marx...).

Aumont et Mesnier (1992) définissent le «sens commun » de médiateur :

[...] le médiateur est celui qui favorise la « négociation » dans un conflit tel que peut le vivre tout apprenant dans une relation parfois difficile à un objet de savoir qui lui résiste et le malmène.

Cette définition reprend du premier sens mentionné par le GRE la notion d'intervention d'un tiers pour faciliter la résolution d'un conflit entre deux parties. Ce point de vue est en résonance avec les pédagogies de l'apprentissage (Altet 1997) qui mettent en avant la notion de «conflit cognitif» et, par ricochet, insistent sur le rôle médiateur de l'enseignant dans la résolution de conflits de ce type. 

terme initial à un terme final » ouvre des perspectives intéressantes. En effet, ces termes pourraient tout aussi bien convenir à la définition de l'activité d'enseignementapprentissage qu'à celle de la médiation pédagogique. Raynal et Rieunier donnent une définition de la médiation qui s'éloigne sensiblement du sens étymologique mentionné plus haut :

Ensemble des aides ou des supports qu'une personne peut offrir à une autre personne en vue de lui rendre plus accessible un savoir quelconque. [...] Le langage, l'affectivité, les produits culturels, les relations ou les normes sociales sont des médiations. [...] L'enseignant est un médiateur. $(1997: 220)^{3}$

Ce point de vue est très proche de celui des "pédagogies de la médiation », exprimé par exemple dans la suite de la définition que donne Avanzini :

Dans le registre de l'éducation, ce concept désigne l'entreprise de celui qui aménage et facilite la mise en rapport de la culture avec un sujet qui a, jusqu'alors, échoué à l'assimiler et à la situation duquel on cherche à remédier (re-médier). [La médiation] ... est indispensable à l'activité d'apprentissage. (op. cit. : 14)

Raynal et Rieunier signalent que - outre le Programme d'Enrichissement Instrumental (PEI) de Feuerstein - ce sont les travaux de Vygotski et de Bruner qui ont principalement contribué à établir la médiation comme facteur décisif du développement cognitif de l'enfant.

\subsection{Vygotski et la médiation}

De Vygotski on cite souvent le concept de « Zone prochaine de Développement (ZPD) » :

La disparité entre l'âge mental, ou niveau présent de développement, qui est déterminé à l'aide des problèmes résolus de manière autonome, et le niveau qu'atteint l'enfant lorsqu'il résout des problèmes non plus tout seul, mais en collaboration détermine précisément la zone prochaine de développement. ([1934] 1997 : 35)

Cette notion de ZPD met en évidence l'importance de la médiation du maître, de la collaboration de l'adulte. Vygotski indique également que la médiation et la collaboration de l'adulte ont des limites. Il ne sert à rien d'apprendre à l'enfant ce que son stade actuel de développement ne lui permet pas d'apprendre. Pour être efficace, l'intervention de l'adulte, du maitre, doit se situer à l'intérieur de la ZPD. Mais la ZPD n'est pas le seul domaine où cet auteur fait appel au paradigme de l'opposition seul/en collaboration, direct/indirect ou encore médiat/immédiat. Dans son étude du développement de la conceptualisation chez l'enfant qui est au coeur de son ouvrage de 1934, Vygotski établit une opposition parallèle entre les notions de concept spontané et de concept scientifique d'une part et celles de rapport immédiat et de rapport médiatisé d'autre part :

La première apparition d'un concept spontané est ordinairement liée à un heurt direct de l'enfant avec telles ou telles choses ... des choses réelles, des choses de la vie. Le concept scientifique, par contre, a pour point de départ non pas un heurt direct avec les choses, mais un rapport médiatisé avec l'objet ${ }^{4}$.

\subsection{Bruner et l'étayage}

19 Après Vygotski, Jerome Bruner, théoricien de l'apprentissage par la découverte, a développé le concept de médiation sous diverses appellations, dont le tutorat et l'étayage (scaffolding), qu'il définit ainsi : 
[Scaffolding] refers to the steps taken to reduce the degrees of freedom in carrying out some task so that the child can concentrate on the difficult skill she is in the process of acquiring. (Bruner 1978, cité par Mercer 1995 : 73) enseignant, il semble bien que toute relation pédagogique doive inclure des actions de médiation, et qu'un enseignant ait nécessairement à jouer un rôle de médiateur. Faut-il pour autant aller jusqu'à établir une équivalence absolue entre enseignement et médiation? Aumont et Mesnier pensent qu'une telle équation serait réductrice :

La fonction d'aide à l'apprentissage ne peut se résumer à une activité de médiation. [...] Le libre accès à des ressources - qu'il s'agisse de pairs engagés dans le même apprentissage (et pouvant aussi jouer un rôle de médiateur), d'experts dans un savoir, de documents ou guides interactifs - constitue un aspect essentiel de toute démarche d'appropriation d'un nouveau savoir. (1992: 207) 
l'aider à résoudre ses conflits cognitifs, ou sous son aspect d'étayage aux côtés de l'apprenant pour le soutenir en attendant qu'il puisse voler de ses propres ailes, la médiation pose toujours problème. Situé dans cette position d'intermédiaire entre l'apprenant et le savoir, au lieu de servir de voie de communication, le maitre peut - dans le cadre des pédagogies centrées sur le contenu - faire barrage. Bien que, dans la métaphore de l'étayage, le maître ne prenne plus position entre le savoir et l'élève, mais aux côtés de ce dernier pour le soutenir, le confort de ce soutien provisoire peut inciter celui-ci à ne plus vouloir ou pouvoir s'en passer. Cette contradiction de la fonction de médiation, nécessaire dans la relation pédagogique, mais condamnée à disparaitre, est exprimée dans la définition qu'en donne Meirieu :

[la] médiation désigne à la fois ce qui, dans le rapport pédagogique, relie le sujet au savoir et sépare le sujet de la situation d'acquisition. Elle assure ainsi, contradictoirement, mais indissolublement, la transmission du savoir et l'émancipation du sujet. (1987: 187)

\subsection{Pour une représentation dynamique de la médiation pédagogique}

Nous allons tenter de trouver une place pour la médiation pédagogique qui puisse s'insérer dans notre deuxième modèle de la situation pédagogique. $\mathrm{Si}$, dans cette situation, le médiateur était toujours et seulement l'enseignant, alors la médiation se confondrait totalement avec notre axe du processus Enseigner. Puisque tel n'est pas le cas, nous proposerons une représentation « dynamique » de la médiation pédagogique.

Figure 4. Les deux « leviers » de la médiation pédagogique

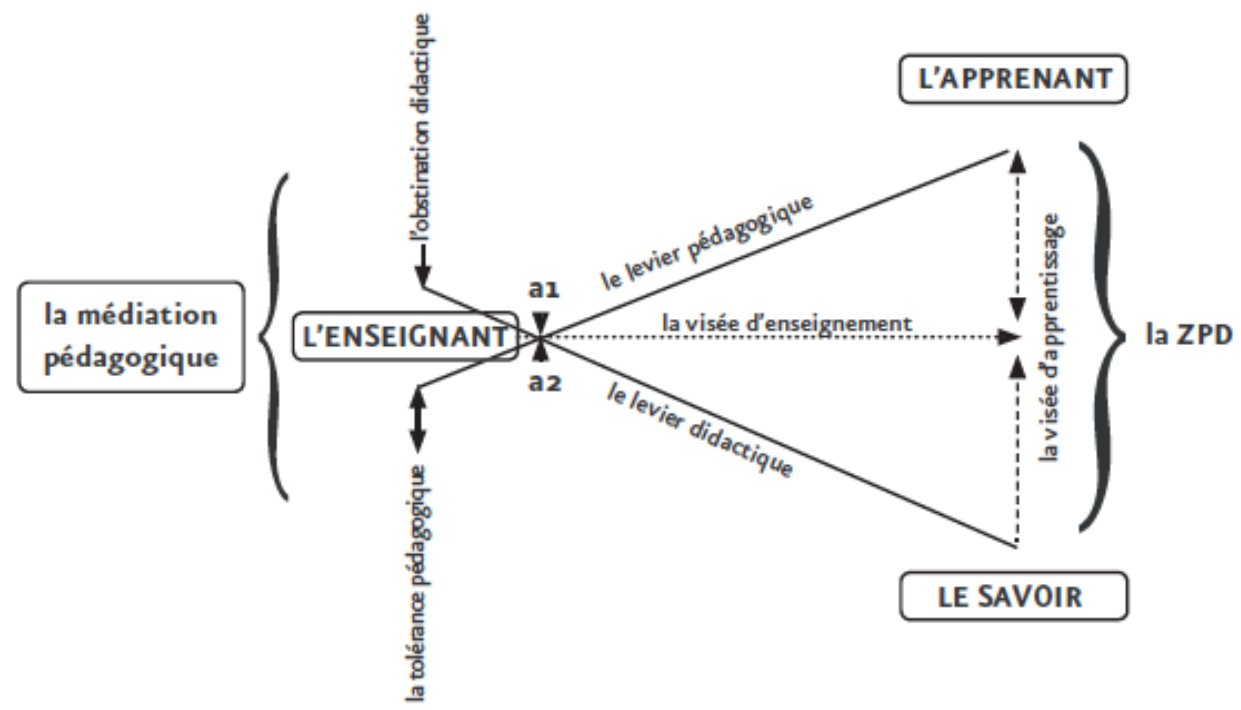



modèle utilise la métaphore du levier (les points d'appui sont a1 et a2). Nous considérons que la médiation pédagogique est toujours la combinaison (en proportions variables), la résultante de l'application de deux forces que nous appellerons (d'après Develay 1992: 13) "l'obstination didactique» et "la tolérance pédagogique ». La première de ces deux forces cherche à rapprocher le savoir de l'apprenant, tandis que la seconde cherche à rapprocher l'apprenant du savoir. La flèche à simple orientation de la «force » didactique symbolise cette obstination qui ne se relâche jamais, tandis que la flèche à double orientation indique que la « force " pédagogique ne peut s'exercer en sens unique, elle doit être souple, élastique, permettre le «jeu», sans quoi le levier pédagogique se rompra et le contact avec l'apprenant sera perdu.

Une telle représentation permet de suivre au plus près l'évolution de la "quantité » de médiation qui alternativement « relie l'apprenant au savoir et le sépare de la situation d'acquisition» (pour reprendre les termes de Meirieu). Elle met l'accent sur une métaphore de l'apprentissage vu comme un "rapprochement» qui s'opère entre l'apprenant et le savoir, rapprochement médiatisé par l'enseignant, à l'intérieur de la ZPD définie par Vygotski. Notre modèle permet en outre de visualiser certaines « dérives » de la relation pédagogique. Si le pôle de l'enseignant «glisse » le long de l'axe que nous avons appelé "la visée d'enseignement", jusqu'à venir occuper la place située entre l'apprenant et le savoir, au lieu de faire office de médiation, l'enseignant devient un obstacle, il fait barrage à l'apprentissage. De même, s'il se produit un déséquilibre entre les forces qui s'appliquent sur les deux « leviers » de la médiation pédagogique, on obtient soit une situation où le savoir a certes été didactisé mais se trouve présenté à un apprenant qui n'a pas été mis en mesure de se l'approprier, soit la situation inverse, dans laquelle un apprenant a bien été mis en situation d'apprentissage, mais se trouve confronté à un savoir non assimilable par lui parce que non-didactisé.

\section{Médiatisation et médiation}

\subsection{Du triangle au carré pédagogique}

Étant donné que notre projet de recherche-action implique l'utilisation des nouvelles technologies, nous nous sommes intéressé au processus de didactisation avec instruments - que nous appellerons médiatisation. Examinons pour commencer le modèle des Situations d'Activités Instrumentées de Rabardel (1985 et 1995). 


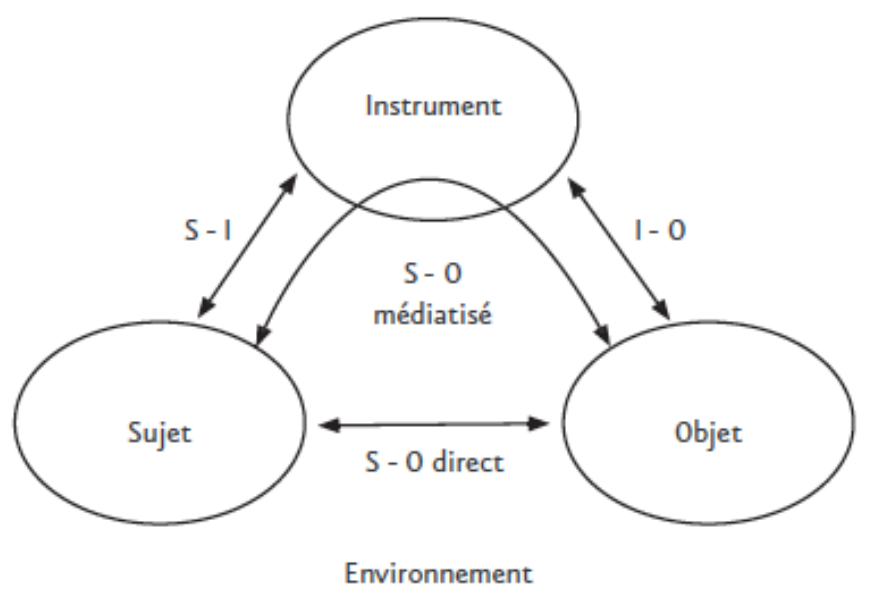

La comparaison entre la situation d'activité avec instruments ${ }_{\text {sujet }}^{\text {inSTRUMment }}$ et la médiateur joué par l'instrument dans la première et par l'enseignant dans la seconde. Dans le cadre d'une situation d'apprentissage avec instruments on pourrait certes envisager de redéfinir le triangle didactique en triangle SAI. C'est ce que propose Raby pour qui, « dans le cadre de l'auto-formation, le triptyque savoir-sujet-enseignant doit se redéfinir en sujet-instrument-savoir (1996: 367)». C'est également la solution adoptée dans le modèle de Legendre revisité par Germain. Cependant, ces modèles présentent l'inconvénient soit de faire disparaître l'enseignant soit de lui attribuer une nature « instrumentale », ce qui nous paraît également inacceptable. Nous préférerons donc dire que, dans la situation d'apprentissage avec instruments, des relations s'établissent entre un objet (le savoir) et non pas un, mais deux sujets (l'enseignant et l'apprenant). Nous serons ainsi amené à modifier à nouveau notre modèle de la situation pédagogique. La théorie de Rabardel va d'ailleurs nous y aider.

Rappelons que, pour cet auteur, l'instrument est une entité bifaciale composée d'une part de l'artefact et d'autre part des schèmes d'utilisation (du concepteur et de l'utilisateur). La "genèse instrumentale ». comporte deux types de processus associés : les processus d'instrumentalisation et les processus d'instrumentation (Rabardel 1995 : 137 sqq.). Nous proposons d'assimiler le travail de didactisation effectué par l'enseignant aux processus d'instrumentalisation qui concernent l'émergence des composantes artefact de l'instrument: «sélection, regroupement, production et institution de fonctions, détournements et catachrèses, attribution de propriétés, transformation de l'artefact ».

Ce travail de didactisation prend place au sein d'une relation

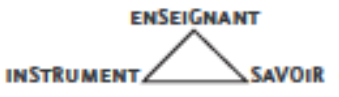
dans laquelle l'enseignant joue un double rôle de médiateur du savoir et de concepteur de l'instrument. Quant aux processus d'instrumentation, ils constituent d'après Rabardel l'autre face de la genèse instrumentale, celle de l'utilisation :

les processus d'instrumentation sont relatifs à l'émergence et à l'évolution des schèmes d'utilisation et d'action instrumentée: constitution, fonctionnement, 
évolution par accommodation, coordination, combinaison, inclusion et assimilation réciproque, assimilation d'artefacts nouveaux à des schèmes déjà constitués [...]. (op. cit. : 137)

On reconnaît là les caractéristiques des processus de l'apprentissage tels que définis, par exemple, par Piaget (accommodation et assimilation)...

Tandis que les processus d'instrumentalisation sont orientés du sujet (S1, Enseignant) vers l'artefact, ceux d'instrumentation sont orientés de l'artefact vers le sujet (S2,

Apprenant). Nous sommes ici dans une relation

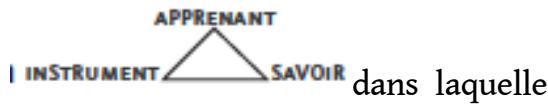
l'apprenant accède au savoir via la médiation de l'instrument. Au moment de combiner en un seul système les deux sous-systèmes que constituent les relations

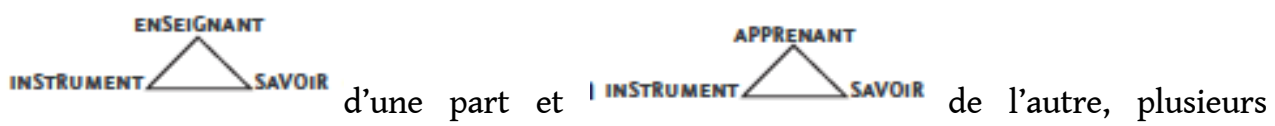
options se présentent à nous. Une version ancienne de notre modèle se présentait sous la forme d'un tétraèdre, ce qui permettait de visualiser et d'étudier les quatre relations triangulaires figurées par les quatre faces de cette fi gure (Rézeau, 1998). Le modèle proposé par Bertin (2001) est également quadripolaire : il s'agit d'un modèle ergonomique global de la situation d'AMO ${ }^{6}$ regroupant deux sous-systèmes. Pour cet auteur, le soussystème apprenant-ordinateur est celui de l'ergonomie cognitive, tandis que le soussystème enseignant-ordinateur relève de l'ergonomie pédagogique. Nous partageons avec Bertin l'insistance sur « la présence de deux pôles humains [qui] sous-entend aussi celle de deux sujets au sein du même processus » (op. cit. : 252), ainsi que les références aux travaux de Rabardel. Au lieu de centrer notre approche sur la notion d'ergonomie, nous préférons toutefois parler de médiation et de médiatisation. C'est d'ailleurs afin d'attribuer à ces deux notions une place centrale dans la situation pédagogique que nous avons abandonné le modèle du tétraèdre pour celui du carré pédagogique. Ce carré est obtenu en réunissant les deux relations triangulaires par leur axe commun, celui qui relie les instruments au savoir. 
Figure 6. Troisième modèle de la situation d'enseignement-apprentissage : le carré pédagogique (version complète)

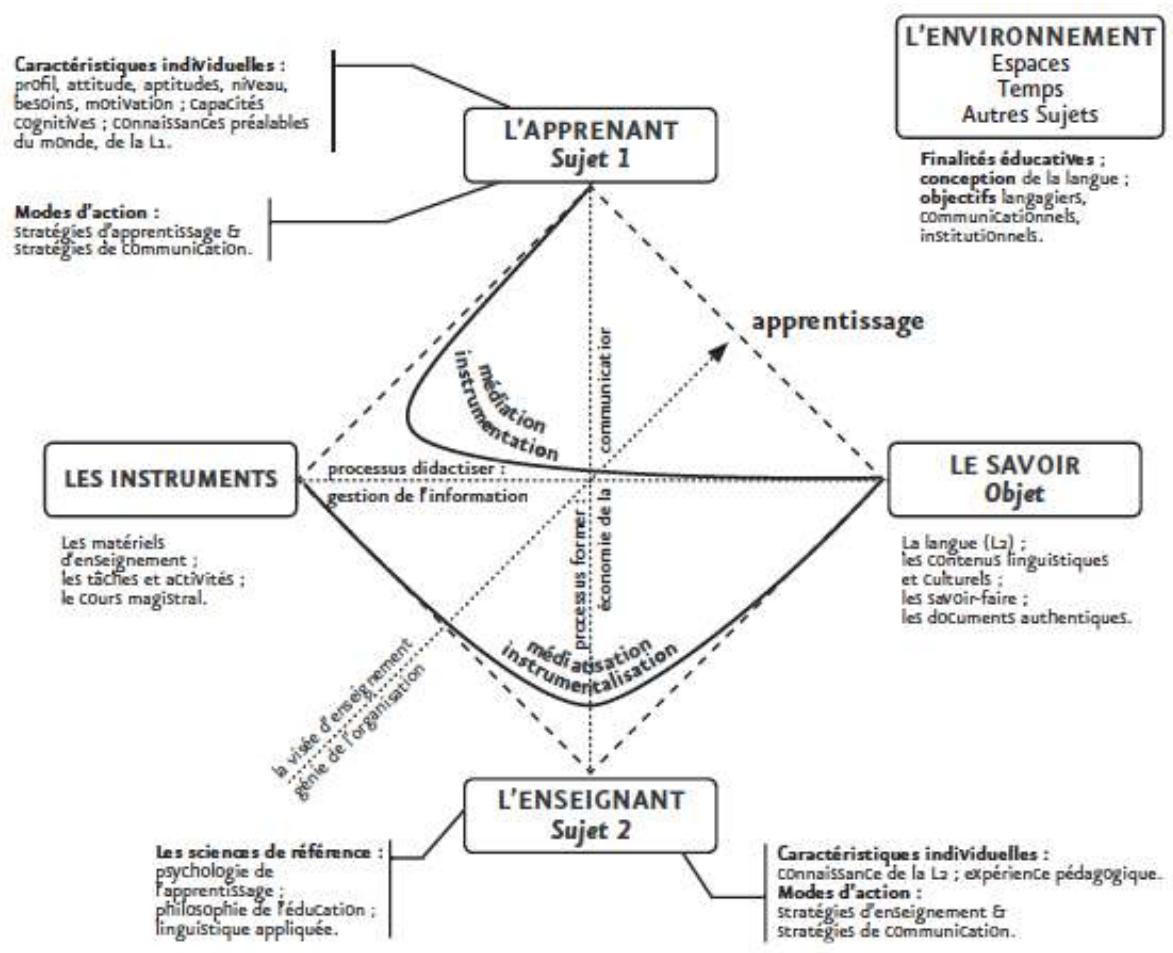

\subsection{Médiation et médiatisation : de nouvelles définitions} entraîner la position d'un élément B placé au milieu entre deux autres éléments A et $C$ ? L'élément B peut jouer un rôle d'intermédiaire (médiation); ce rôle d'intermédiaire peut être positif (conciliation); il peut être négatif (faire barrage), ou simplement neutre (remplacer une relation directe entre A et $C$ par une relation indirecte) ; l'élément $B$ peut être un moyen pour A d'atteindre C (ou vice-versa). Dans le cadre de la situation pédagogique, $A$ étant l'apprenant et $C$ le savoir, nous proposons d'attribuer à l'élément $B$ les rôles et les définitions qui suivent. 
Dans le domaine de la Didactique des Langues Étrangères (DLE) nous proposons la définition suivante.

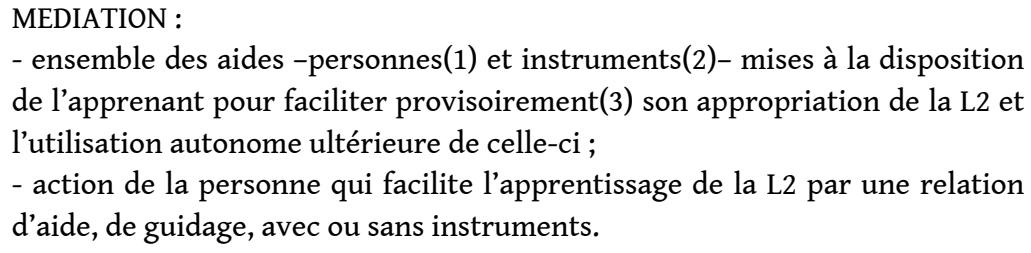

NOTES :

1. Ces personnes, enseignant, formateur, tuteur, camarade, l'apprenant lui-même, etc. sont des médiateurs.

2. Il s'agit des instruments tels que définis par Rabardel (1995). En référence à cette théorie, la médiation correspond aux processus d'instrumentation, qui sont relatifs à l'émergence et à l'évolution des schèmes d'utilisation et d'action instrumentée.

3. En référence aux théories sociocognitives de la médiation sociale (Vygotski, Bruner), l'étayage facilitateur de la médiation est une étape nécessairement provisoire. De même, en référence à l'emploi général de médiation, «entremise destinée à mettre d'accord, à concilier ou à réconcilier des personnes, des partis ", lorsque les parties sont réconciliées, l'entremise n'a plus d'objet.

37 La double définition met en évidence le fait que la médiation peut être le processus résultant soit de l'action directe d'une personne soit de l'action indirecte exercée à travers des instruments.

En ce qui concerne la médiatisation, nous avons souhaité utiliser ce terme pour remplacer celui de didactisation, pour plusieurs raisons. Il s'agit tout d'abord d'un terme relativement peu utilisé dans le domaine de la didactique en général comme dans celui de la DLE; on lui préfère la notion de transposition didactique. Mais, dans le cadre de la DLE, cette notion de "transposition du savoir savant en savoir à enseigner " semble moins pertinente que dans le cadre d'autres disciplines scolaires qui ont effectivement à transmettre un savoir plutôt qu'un savoir-faire. Pour composer le sens attribué ici à médiatisation, nous avons emprunté d'une part à la première entrée du mot dans le Nouveau Petit Robert, emploi 2: "rendre médiat » (c'est-à-dire introduire un élément intermédiaire, une distanciation, une didactisation) et d'autre part à la seconde entrée ce qui, dans la filiation étymologique remonte à l'idée de média, de moyens, c'est-à-dire « ce qui sert pour arriver à une fin ». Nous avons également incorporé à cette définition des éléments de la théorie de la genèse instrumentale de Rabardel, ce qui donne :

MEDIATISATION : opération consistant à sélectionner, transformer, transposer les matériaux linguistiques et culturels de la L2 pour en faire des instruments(1) (contenus, méthodes, matériels, tâches) répondant à des besoins pédagogiques déterminés(2).

1. La médiatisation correspond ici aux processus d'instrumentalisation, qui concernent l'émergence des composantes artefact de l'instrument: "sélection, regroupement, production et institution de fonctions, détournements et catachrèses, attribution de propriétés, transformation de l'artefact » (Rabardel 1995).

2. L'opération de médiatisation supposant des compétences et des moyens matériels qui ne sont pas habituellement accessibles à l'apprenant ou à ses pairs, la personne qui réalisera ce type d'opération sera un enseignant, un formateur. Dans le cas où cette opération a pour but la création de matériels d'enseignement, elle pourra être conduite par un concepteur qui ne sera pas nécessairement un enseignant. Nous appellerons cette personne un médiatisateur. 
Il subsiste un problème terminologique: en effet, aux substantifs médiation et médiatisation ne correspond qu'un seul et même verbe, médiatiser. Pour désigner le processus de médiation, il nous semblerait préférable d'utiliser le néologisme médier, qui commence à faire son apparition dans la littérature du domaine ${ }^{7}$.

\section{Instruments et nouvelle médiation pédagogique}

41 Après avoir redéfini la place de l'enseignement dans le triangle pédagogique, nous avons proposé de transformer celui-ci en carré pédagogique. Ce nouveau modèle nous a permis d'attribuer aux instruments une place à part entière en tant qu'agents médiateurs du savoir. Il nous a également permis de définir et de placer, chacune à la place qui lui revient, la médiation-instrumentation et la médiatisation-instrumentalisation. Dans ce quatrième et dernier point, il nous faut évoquer le nouveau rôle dévolu à l'enseignant dans une situation d'apprentissage avec instruments. Les notions de transparence et d'opacité associées aux instruments nous permettront d'évoquer des problèmes classiques en DLE. Nous verrons enfin comment il est possible de concilier le rôle médiatisateur de l'enseignant avec le rôle médiateur de l'instrument.

\subsection{Rôle de l'enseignant et rôles de l'ordinateur}

Que l'on se place dans la perspective du tutoriel traditionnel - héritier direct de l'enseignement programmé - ou dans celle du «tutoriel sophistiqué » tel que conçu et mis en œuvre par Demaizière (1986), la question se pose du rôle dévolu à l'enseignant dans une situation d'apprentissage avec ordinateur. Cette question n'est pas nouvelle ni propre à l'EAO: elle surgit inévitablement dès que l'on passe du paradigme des pédagogies magistrales à celui des pédagogies centrées sur l'apprenant ou sur l'apprentissage. Dans les pédagogies de l'apprentissage, certains auteurs considèrent l'enseignant comme « un adjuvant humain, parmi d'autres adjuvants, [...] matériels et technologiques » (Springer 1996: 166). Mais il semble bien qu'avec l'ordinateur tuteur la présence d'un professeur «virtuel » dans la machine évince pour de bon le professeur réel, l'adjuvant technologique étant devenu un substitut de l'enseignant.

Un argument constamment mis en avant par les promoteurs de l'ordinateur tuteur est celui de la libération de l'enseignant pour d'autres tâches, plus gratifiantes, et non mécanisables. La vision de l'ordinateur tuteur comme incarnation du «caractère mécanique de la fonction du maître telle que la conçoit l'enseignement traditionnel » (Piaget 1969 [1965] : 107) nous semble condamnable et condamnée précisément parce qu'elle renvoie à une conception dépassée de l'enseignement.

Si l'on s'oppose à cette vision, deux options, semble-t-il, restent à l'enseignant qui veut continuer à jouer un rôle de médiateur dans la situation pédagogique: une option maximaliste dans laquelle le professeur, créant ses propres tutoriels, se met lui-même en machine; une option minimaliste qui conduit à utiliser l'ordinateur comme un outil. S'agissant de la première option, celle de la médiatisation du savoir, deux points de vue s'opposent : les « professionnels » de l'ingénierie didactique rejettent le « bidouillage » ou bricolage et prônent la séparation des tâches (cf. Demaizière 1986: 74). Nous ne partageons pas ce point de vue, mais plutôt celui de Linard qui écrit : 
Si la qualité de la médiatisation technique de l'apprentissage ne vaut que ce que vaut la médiation des hommes qui l'interprètent et la font vivre sur le terrain, alors il faut sortir de la division du travail entre les concepteurs et les exécutants de l'innovation qui prévaut encore dans la plupart des systèmes éducatifs. (1996: 116)

Un paradigme classique dans le domaine de l'EAO est celui qui oppose pour l'ordinateur un rôle de tuteur à celui d'outil. Le premier rôle renvoie aux machines à apprendre de Skinner, le second rôle, plus spécialement en langues, renvoie à l'approche communicative. Entre outil, instrument et machine, quelle analogie serait la mieux susceptible de rendre compte de la nature protéiforme de l'ordinateur? Considéré en tant que machine à apprendre, l'ordinateur présente à la fois les caractéristiques d'un outil et celles d'un instrument ${ }^{8}$. Ces deux aspects sont toujours présents, mais plus ou moins visibles selon l'usage qui est fait de la machine ordinateur.

Le paradigme de l'ordinateur outil met l'accent sur un rôle prosthétique permettant de pallier des déficiences humaines, sur la médiation pragmatique qui vise à renforcer l'action humaine. Les représentations correspondant à ce paradigme sont celles qui considèrent l'ordinateur comme "un simple outil», incapable d'initiative propre, mais utile pour améliorer l'efficacité de l'homme. En outre, l'idée que l'ordinateur peut améliorer quantitativement l'enseignement et l'apprentissage rejoint les thèses déjà anciennes de la mécanisation de l'enseignement (cf. Skinner) ou celles, actuelles, de l'industrialisation de la formation (cf. Alberganti 2000).

Le paradigme de l'ordinateur instrument n'est pas mentionné directement dans la littérature des NTIC, ${ }^{9}$ mais on peut considérer que les paradigmes de l'outil cognitif ou des environnements informatiques d'apprentissage humain peuvent lui être assimilés. Dans ces paradigmes, l'accent est mis sur la médiation épistémique de l'instrument qui permet une meilleure perception et une meilleure connaissance de l'objet d'étude ${ }^{10}$.

Dans leur étude de l'évolution des conceptions du rôle de l'ordinateur en EAO, la plupart des auteurs du domaine font mention d'un abandon du paradigme de l'ordinateur-tuteur au profit du paradigme de l'ordinateur-outil. Selon Wolff, par exemple,

...in learner autonomy, the key concept in modern language learning and in all humanistically oriented pedagogy, the computer as tutor cannot have a place. Making use of its tool functions, on the other hand, can support learning in a variety of ways. (1999: 128)

Étant donné la multitude de fonctions et d'usages de l'ordinateur, ne devrait-on pas plutôt le considérer sinon comme un outil à tout faire (a general-purpose tool) du moins comme un outil à usages multiples (a multi-purpose tool) ? C'est le point de vue adopté par Martin qui considère que, avec l'arrivée des NTIC, on assiste à une mutation radicale de l'OT [objet technique] qui passe du statut de simple objet façonné pour l'usage à celui d'outil de prospection. Désormais, l'OT n'est plus (seulement) le moyen de mettre en relation un projet avec une finalité dans la mesure où il n'y a plus de finalité définissable, mais une multifonctionnalité [...]. $(2001: 34)^{11}$

En mettant ainsi l'accent sur la valeur de médiation épistémique du «nouvel OT », et en l'opposant corrélativement à la valeur de médiation pragmatique du «simple outil » ancien, cet auteur semble rejoindre notre proposition selon laquelle l'ordinateur est davantage un instrument qu'un outil. 


\subsection{Transparence et opacité}

51 Une notion intéressante lorsqu'on étudie la fonction médiatrice des outils (cognitifs) est celle de transparence (et de son corollaire l'opacité). Considérons les citations suivantes :

L'artefact est un médiateur dont la présence ne doit faire en aucune manière obstacle à la relation du sujet à l'objet de son activité. (Rabardel 1995 : 184)

... une bonne interface est celle qui disparaît cognitivement lors de l'usage de l'outil et avec laquelle nous n'avons affaire que lors de situations inattendues ... la nécessité de « visibilité » est variable. (Polanyi, cité par Rabardel op. cit. : 185)

For most routine purposes, awareness of a medium may hamper its effectiveness as a means to an end. Indeed, it is typically when the medium acquires transparency that its potential to fulfi l its primary function is greatest. (Chandler $1995: 10$ )

Tous ces auteurs affirment que l'efficacité des artefacts, instruments ou média est directement proportionnelle à leur transparence. Le détour par les instruments nous ramène ainsi à un thème central en DLE, celui qui regroupe les divers concepts de conceptualisation («language awareness »), de métacognition, de l'explicite par rapport à l'implicite. Pour reprendre sous un autre éclairage le débat classique entre les approches communicative et conceptualisatrice en enseignement des langues, on pourrait dire que la première recherche une transparence maximale tandis que la deuxième admet la nécessité d'une certaine "opacité » ou plus précisément d'une certaine visibilité. Mais, peut-on se demander, qu'est-ce exactement qui peut être visible ou transparent dans l'apprentissage et l'utilisation d'une langue? On sait que l'option constructiviste de la psychologie cognitive postule que la réalité est toujours une construction par l'être humain. Chandler (déjà cité) évoque deux types de comportement face à ce postulat :

Romantics construct the 'natural' in attempting to stay close to experience through 'immediacy' and 'authenticity'. Objectivists construct 'facts', attempting to view the world without 'distortion' as if through a transparent 'window'. Both seek to escape mediation. (op. cit. : 32 )

Si nous appliquons la grille de lecture proposée par Chandler aux conceptions en DLE, les « romantiques » seraient les tenants de la méthode directe, de l'approche communicative, du «bain linguistique » et d'un certain nombre de méthodes humanistes. De même que les romantiques prônaient un retour vers l'état de nature et l'état d'enfance, ces différentes conceptions de l'apprentissage des langues mettent en avant ce qui rapproche l'apprentissage de la L2 de celui de la L1; le concept d'authenticité fait également partie de leur credo. À l'opposé, les « objectivistes » en DLE seraient les tenants des pédagogies du contenu ainsi que les behavioristes. On peut considérer que ces différentes approches cherchent, chacune à leur manière et à des degrés divers, à établir entre l'apprenant et la L2 un rapport direct, immédiat. Elles rejettent par conséquent l'apport de toute médiation au processus de construction de la réalité de cette L2. En revanche, les didacticiens des langues qui s'appuient sur l'apport du constructivisme savent bien qu'il n'y a ni perception immaculée ni apprentissage sans risques. Pour eux, "comprendre le système aide l'élève à comprendre la langue » (Bailly 1997 : 103). La médiation envisagée ici est multiple : médiation par les outils cognitifs (conceptualisation et métacognition), médiation de l'enseignant, médiation par les pairs. Dans tous les cas de figure il s'agit bien de rendre visible l'invisible (Richterich 1996) et donc de procurer à l'apprenant tous les instruments et médias nécessaires pour mieux percevoir et construire la réalité de la L2. 


\section{3. « Néo-cognitivisme » : la cognition distribuée} soviétiques du développement, plusieurs auteurs ont proposé de redéfinir la cognition comme « un processus distribué à travers les objets, les outils et les artefacts propres à une culture » (Depover I. 1998 : 163). La grande nouveauté de la cognition distribuée c'est d'introduire, avec la dimension écologique, non seulement les autres humains, mais aussi les outils. Or, avec l'émergence des nouvelles technologies, les outils sont encore plus proches de l'homme, plus proches de la cognition et de l'intelligence humaine. Parce qu'il est davantage comparable à un clavecin qu'à un marteau, parce que - en tant qu'instrument - il est le vecteur d'une médiation épistémique tout autant que pragmatique, l'ordinateur peut sans aucun doute jouer un rôle d'acteur dans l'intelligence collective. Comme l'écrit Lévy dans son ouvrage consacré aux technologies de l'intelligence :

Les dispositifs techniques sont donc bien des acteurs à part entière dans un collectif qu'on ne peut plus dire purement humain, mais dont la frontière est en redéfinition permanente. $(1990: 157)$ scientifique, Laurillard distingue les connaissances de premier ordre qui concernent notre expérience « immédiate » du monde (principalement fondée sur la perception) de celles de second ordre qui supposent une prise de recul, une médiation, une «métaexpérience » :

Everyday knowledge is located in our experience of the world. Academic knowledge is located in our experience of our experience of the world. [...] Thus teaching is [...] mediated learning, allowing students to acquire knowledge of someone else's way of experiencing the world. (1993:26)

Cette auteure indique donc clairement que le rôle de l'enseignant est celui d'un médiateur de l'apprentissage. Elle en arrive à la conclusion que le processus d'enseignement-apprentissage idéal est un dialogue de type socratique entre le professeur et l'étudiant. Étant donné qu'il n'est matériellement pas possible d'avoir un professeur pour chaque étudiant, pourquoi ne pas recourir aux Nouvelles Technologies? Le tutoriel intelligent serait la solution idéale, mais elle affirme qu'il n'existe pas: "Tutoring systems would be the acme of all the educational media, if they existed.» (1993: 29) La réponse est sans doute de ne pas croire que l'ordinateur suffise, seul et par lui-même, à provoquer acquisition ou apprentissage, et donc à remplacer l'enseignant. Pour décrire ce rôle de médiateur auxiliaire, Salomon [1995] utilise la métaphore du levain dans la pâte.

\section{Conclusion : réconcilier médiation et médiatisation}

Monique Linard (1996) associe d'une part le cognitivisme computationnel avec une conception technocentrique et prosthétique des instruments (la médiatisation), et de l'autre la cognition vue comme "activité de connaissance" avec une conception anthropocentrique des outils cognitifs (la médiation). Nous ne partageons pas ce point de vue qui établit une équation entre la médiatisation et la seule perspective technocentrique du paradigme du «cognitivisme informationnel» et qui restreint 
corrélativement l'emploi du terme de médiation au domaine du constructivisme « existentiel ». Pour nous, dans la situation pédagogique, médiatisation et médiation sont dans le même rapport bifacial que les processus d'instrumentalisation et d'instrumentation dans la genèse instrumentale. Linard reconnaît d'ailleurs que

L'outil n'est pas un simple objet. À la fois intermédiaire de connaissance et moyen de capitalisation sociale de l'expérience, il devient 'médiateur' au sens de tiers actif dans les relations entre sujets et objets. (op. cit. : 262)

Or, si l'on veut reconnaître à l'instrument un rôle essentiel de médiateur dans l'action et l'apprentissage, il faut bien dans le même temps reconnaître l'importance corrélative de l'activité de médiatisation - sans laquelle l'instrument n'existerait pas.

61 Nous avons postulé dans la première partie de cet article que l'apprentissage n'est pas une mise en relation simple et directe entre l'apprenant et le savoir, mais qu'il résulte nécessairement d'une visée d'enseignement. De la même manière, nous proposons que dans le cadre d'un apprentissage avec instruments - il ne saurait y avoir médiation sans médiatisation.

\section{BIBLIOGRAPHIE}

Alberganti, Michel. 2000. À l'école des robots ?, L'informatique, l'école et vos enfants. Paris : CalmanLévy.

Altet, Marguerite. 1997. Les pédagogies de l'apprentissage. Paris : Presses Universitaires de France.

Astolfi , Jean-Pierre. 1997. « Du 'tout' didactique au 'plus' didactique ». Revue française de pédagogie 120, 67-73.

Aumont, Bernadette et Pierre-Marie Mesnier. 1992. L'acte d'apprendre. Paris : Presses Universitaires de France.

Avanzini, Guy. 1996. « Petit Lexique ». In Médiation éducative et éducabilité cognitive. Lyon : Chronique Sociale.

Bailly, Danielle. 1997. Didactique de l'anglais (1) Objectifs et contenus de l'enseignement. Paris : Nathan.

Bertin, Jean-Claude. 2001. «L'ergonomie didactique : une approche de la recherche dans le domaine de l'apprentissage médiatisé par ordinateur ». In Mémet M. et M. Petit (dir.), L'anglais de spécialité en France. Mélanges en l'honneur de Michel Perrin. Bordeaux : GERAS Éditeur, 237-254.

Bruner, Jerome S. 1983. Le développement de l'enfant, savoir faire, savoir dire. Paris : PUF [Traductions d'articles parus entre 1970 et 1976].

Chandler, Daniel. 1995. The Act of Writing, A Media Theory Approach. Aberystwyth: University of Wales. Juin 2001 http://users.aber.ac.uk/dgc/act.html.

Demaizière, Françoise. 1986. L'enseignement assisté par ordinateur. Paris : Ophrys.

Depover, Christian, Max Giardina et Philippe Marton. 1998. Les environnements d'apprentissage multimédia, Analyse et conception. Paris : L'Harmattan.

Develay, Michel. 1992. De l'apprentissage à l'enseignement. Pour une épistémologie scolaire. Paris : ESF. 
Germain, Claude. 1989. « Un cadre conceptuel pour la didactique des langues ». Études de linguistique appliquée 75, 61-77.

Houssaye, Jean. 1988. Le triangle pédagogique. Berne : Peter Lang.

Laurillard, Diana. 1993. Rethinking University Teaching, A framework for the effective use of educational technology. Londres \& New York : Routledge.

Legendre, R. 1988. Dictionnaire actuel de l'éducation. Paris \& Montréal : Larousse.

Lerbet, Georges. 1984. « Approche systémique et sciences de l'éducation ». Revue française de pédagogie 67, 29-36.

Lévy, Pierre. 1990. Les technologies de l'intelligence : l'avenir de la pensée à l'ère informatique. Paris : Éditions La Découverte.

Linard, Monique. 1996. Des machines et des hommes, Apprendre avec les nouvelles technologies. Paris \& Montréal : L'Harmattan.

Linard, Monique. 1998.« L'écran de TIC, 'dispositif' d'interaction et d'apprentissage : la conception des interfaces à la lumière des théories de l'action ». Juin 2001 http://hal.archivesouvertes.fr/edutice-00000913/.

Martin, Jacky. 2001. «L'objet technique et ses discours ». In Mémet et Petit (dir.), L'anglais de spécialité en France. Mélanges en l'honneur de Michel Perrin. Bordeaux : GERAS Éditeur, 25-36.

Matthey, Marinette. 1996. Apprentissage d'une langue et interaction verbale. Bern : Peter Lang.

Meirieu, Philippe. 1987. Apprendre ... oui, mais comment. Paris : ESF.

Mémet, Monique et Michel Petit (dir.). 2001. L'anglais de spécialité en France. Mélanges en l'honneur de Michel Perrin. Bordeaux : GERAS Éditeur.

Mercer, Neil. 1995. The Guided Construction of Knowledge. Clevedon, UK : Multilingual Matters.

Piaget, Jean. 1969. Psychologie et pédagogie. Paris : Denoël.

Polanyi, M. 1958. Personal knowledge. Londres : Routledge \& Keagan.

Rabardel, Pierre. 1995. Les hommes et les technologies, Approche cognitive des instruments contemporains. Paris : Armand Colin.

Raby, Françoise. 1996. « Apprentissage de l'anglais et nouvelles technologies éducatives : contribution de l'ergonomie cognitive à la didactique des langues ». Thèse de doctorat de l'Université Victor-Segalen Bordeaux 2.

Raynal, Françoise et Alain Rieunier. 1997. Pédagogie : dictionnaire des concepts clés. Paris : ESF.

Rézeau, Joseph. 1998. « L'apprenant, l'enseignant et la machine : triangle d'or ou triangle des Bermudes? ». Cahiers de l'APLIUT 17/3, 91-104.

Richterich, René. 1996. « La compétence stratégique : acquérir des stratégies d'apprentissage et de communication ». In Holec, Little et Richterich, Stratégies dans l'apprentissage et l'usage des langues. Strasbourg : Conseil de l'Europe, 41-76.

Sabiron, Jean. 1995. «Langue anglaise et étudiants scientifiques ». Thèse de doctorat de l'université Victor Segalen Bordeaux 2.

Salomon, Gavriel. 1995. « What does the design of effective CSCL require and how do we study its effects?' ». Février 2001 http://portal.acm.org/citation.cfm?

$\mathrm{id}=130909 \& \mathrm{dl}=$ GUIDE $\&$ coll=GUIDE $\&$ CFID=79929699\&CFTOKEN=71215064. 
Skinner, B. F. 1968. The Technology of Teaching. New York : Appleton Century Crofts. Trad. Française 1968 : La révolution scientifique de l'enseignement. Bruxelles : Dessart.

Springer, Claude. 1996. La didactique des langues face aux défis de la formation des adultes. Paris : Ophrys.

Vygotski, Lev. 1997 [1934 édition originale en russe]. Pensée et langage. Paris : La Dispute.

Wolff, Dieter. 1999. « Levy, computer-assisted language learning, context and conceptualization ». System 27/1, 125-128.

\section{NOTES}

1. LANgues pour Spécialistes d'Autres Disciplines

2. Thèse consultable en ligne à http://edutice.archives-ouvertes.fr/edutice-00000222.

3. Souligné par les auteurs

4. C'est nous qui soulignons.

5. Rabardel 1995 : 66, Figure 4, «Modèle SAI : la triade caractéristique des Situations d'Activités Instrumentées (d'après Rabardel \& Vérillon 1985)».

6. Apprentissage Médiatisé par Ordinateur

7. Cf. par exemple Rachel Panckhurst, 1997, «La communication 'médiatisée' par ordinateur ou la communication 'médiée' par ordinateur?», Terminologies nouvelles 17, 56-58.

8. Pour Simondon, « on entend par outil l'objet technique qui permet de prolonger et d'armer le corps pour accomplir un geste, et par instrument l'objet technique qui permet de prolonger et d'adapter le corps pour obtenir une meilleure perception ; l'instrument est outil de perception. » Simondon, Gilbert. Du mode d'existence des objets techniques, Paris: Aubier, 1969. C'est nous qui soulignons.

9. Nouvelles Technologies de l'Information et de la Communication. Nous utilisons ce sigle faute de mieux.

10. Nous empruntons les notions de médiation pragmatique et épistémique à Rabardel (op. cit. : 90).

11. C'est nous qui soulignons.

\section{RÉSUMÉS}

Cet article résume une démarche de réflexion théorique sur la place de la médiation pédagogique et de la médiatisation didactique dans le cadre d'un enseignement des langues avec instruments. Il s'agit de réhabiliter le processus Enseigner dans le triangle pédagogique, de placer la médiation au cœur de la relation pédagogique et enfin de redéfinir - pour les réconcilier - les rôles médiateur et médiatisateur de l'enseignant.

This article presents the summary of a theoretical research concerned with the place and role of mediation and "mediatization" within the framework of a multimedia-based teaching of languages. Our aims are to rehabilitate the place of Teaching in the pedagogic triangle, to put 
mediation at the heart of the pedagogic relation and finally to re-define and reconcile the roles of the teacher as a mediator and "mediatizator".

INDEX

Keywords : cognition (distributed), cognition (situated), instrument, mediation, mediatization, tool, triangle (pedagogical)

Mots-clés : cognition (distribuée), cognition (située), instrument, médiation, médiatisation, outil, triangle pédagogique

\section{AUTEUR}

JOSEPH RÉZEAU 